\title{
SOLID-SURFACE LUMINESCENCE ANALYSIS
}

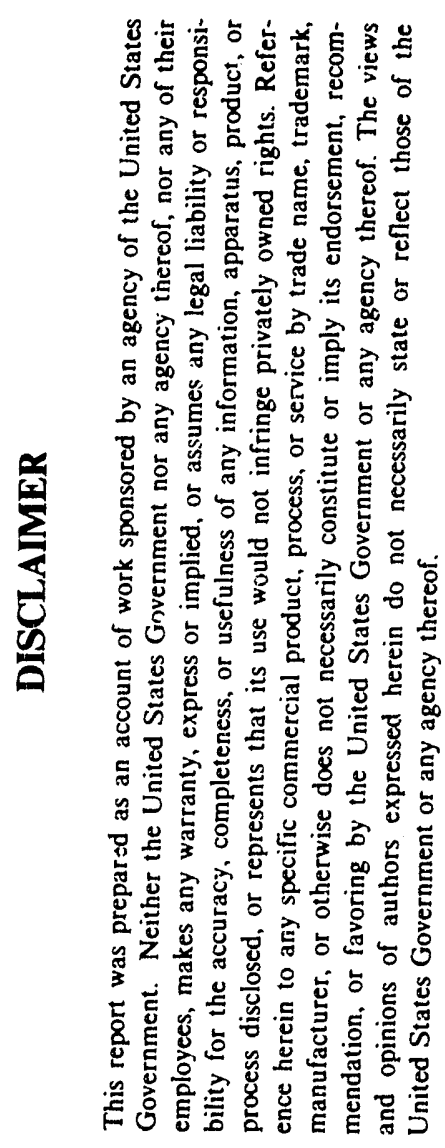

Principal Investigator

Robert J. Hurtubise

Professor

307-766-6241

Proot I How ontro

Progress Report

Period of Grant: 15 June 89-14 June 92

Period of Report: 15 June $89-31$ October 91

R. J. Hurtubise

Chemistry Department

University of Wyoming

Laramie, WY 82071-3838

Prepared for The U.S. Department of Energy

Agreement No. DE-FG02-86ER13547

Department Head

Institutional Administrative Officer

David A. Jaeger

Professor

307-766-2434

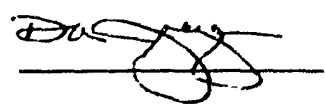

$1 / / 26 / 41$
Dan Baccari

Vice President for Finance

307-766-3306

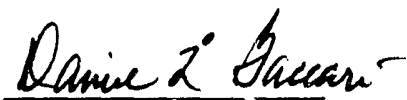

12.3 .91

Date 


\section{Table of Contents}

Page

Summary
A. Introduction
B. Mechanistic Aspects for Lumiphors
C. Interactions with Sodium Acetate
D. Interactions with Filter Paper
E. Interactions with $\beta$-Cyclodextrin $/ \mathrm{NaCl}$ Matrices
F. Analytical Figures of Merit and Analytical Methods of Development
G. Reversed-Phase Liquid Chromatography with $\beta$-Cyclodextrin as a Mobile-Phase Modifier

1

3

6

8

H. Manuscripts and Presentations 


\section{SUMMARY}

In general, this funding period can be characterized as the elucidation of several interactions that are very important in solid-matrix luminescence. With silica gel chromatoplates and filter paper, simple equations were derived for calculating the individual contributions to the percent decrease in luminescence due to either moisture or to a quenching gas. For sodium acetate as a solid matrix and p-aminobenzoate as a model compound, it was concluded that p-aminobenzoate was incorporated into the crystal structure of sodium acetate, and the triplet energy was lost by skeletal vibrations in sodium acetate. Also, with the same system it was shown that p-aminobenzoate did not undergo rotational relaxation, and thus rotational processes did not contribute to the deactivation of the triplet state.

Several results were obtained from model compounds adsorbed on filter paper under different temperature and humidity conditions and with a variety of heavy atoms present. Fundamental photophysical equations were used in calculating several basic parameters that revealed information on rate processes and how the absorbed energy was distributed in an adsorbed lumiphor. The most important advancement with filter paper was the development of equations that relate phosphorescence parameters of adsorbed phosphors to the Young's modulus of filter paper. These equations are based on a fundamental theory that relates the hydrogenbonding network of paper to the modulus of paper.

For cyclodextrin/salt matrices, numerous photophysical parameters were calculate. Phosphorescence polarization daia were obtained as a function of temperature for the first time, 
which permitted the calculation of triplet-state rotational relaxation times. The rotational relaxation times were related to activation energies involved in the nonradiative transition from the triplet state. It was also shown that phosphors had high quantum yields on cyclodextrin/salt matrices, and in one case the phosphorescence quantum yield was the same at room temperature as at $93 \mathrm{~K}$.

Several analytical figures of merit were obtained and analytical methods developed for model compounds adsorbed on solid matrices to illustrate the applicability of solid-matrix luminescence. In addition, the effects of $\beta$-cyclodextrin as a mobile phase additive on the retention characteristics of solutes in reversed-phase liquid chromatography were explored. 


\section{A. Introduction}

In this report, the significant results and conclusions of the research over the last two years and five months will be considered. A variety of the physicochemical interactions that are relevant for obtaining room-temperature fluorescence (RTF) and room-temperature phosphorescence (RTP) from organic compounds adsorbed on solid matrices will be discussed. In addition, the various analytical methods and techniques developed will be presented.

\section{B. Mechanistic Aspects for Lumiphors Adsorbed on Silica Gel with Humidified Gases}

In previous DOE reports, considerable detail was presented on many of the interactions for the RTF and RTP of compounds adsorbed on silica gel chromatoplates. During this funding period, the solid-matrix RTF and RTP data from benzo[f]quinoline (B[f]Q) adsorbed on silica gel chromatoplates in the presence of different humidities and in $\mathrm{N}_{2}$, air, and $\mathrm{O}_{2}$ were interpreted. Simple equations were developed for calculating the individual contributions to the percent decrease in luminescence due to either moisture or to a quenching gas. Thus, a detailed assessment could be made about the quenching of moisture and individual quenching gases on the solid-matrix fluorescence and phosphorescence. Also, based on an isotherm for water uptake by silica gel and the RTP data for B[f]Q, it was concluded that the phosphor molecules near the surface of silica gel are quenched in the range of approximately 0 to $40 \%$ relative humidity and then from $50 \%$ relative humidity to about $75 \%$ relative humidity the phosphor molecules in the pores of the silica gel are quenched (1).

\section{Interactions with Sodium Acetate}

Sodium acetate was used previously as a solid matrix for obtaining RTF and RTP from adsorbed organic compounds. Several interactions important for RTF and RTP were reported 
earlier $(2,3)$. In this work for one set of experiments, the physicochemical interactions of the isomers of aminobenzoic acid were explored (4). RTF and RTP quantum yields of the isomers of aminobenzoic acid adsorbed on sodium acetate were obtained. These data were correlated with solution fluorescence data and diffuse reflectance infrared data from the three isomers. In particular, the specific structural and bonding characteristics of the isomers with sodium acetate were very important in determining whether a high RTP yield would be obtained from a given isomer. The results of these experiments have important analytical implications for fitting nanogram amounts, or less, of phosphors into host crystal structures to obtain selective luminescence signals from guest molecules.

A study was performed to compare solid-matrix luminescence interactions of p-aminobenzoate on filter paper and sodium acetate (5). In this section of the report, only the results from sodium acetate will be discussed. The results for filter paper will be considered in the section on filter paper. With p-aminobenzoate adsorbed on sodium acetate, it was discovered that there was a simple relationship between the reciprocal of $\ln$ [phosphorescence lifetime] as the temperature changed and the reciprocal of the heat capacity of sodium acetate. It was concluded that $p$-aminobenzoate was incorporated into the crystal structure of sodium acetate and the triplet energy was most likely lost via skeletal vibrations in sodium acetate. This is a very important conclusion because it shows for the first time that the RTP of a phosphor incorporated into a crystalline matrix can be related to the vibrational modes of the matrix.

In recent work, solid-matrix luminescence polarization values for p-aminobenzoate adsorbed on sodium acetate and sodium acetate/sodium chloride mixtures were acquired over the temperature range of 296 to $93 \mathrm{~K}(6)$. Interestingly, the fluorescence and phosphorescence polarization values were essentially constant with temperature. These data indicated that 
p-aminobenzoate did not undergo rotational relaxation over the temperature range investigated. In particular, for the p-aminobenzoate in the triplet state, the lack of rotational relaxation indicated that mainly vibrational processes in sodium acetate were responsible for the decrease in phosphorescence quantum yields as the temperature increased. These results are important because they support the concept that the three-dimensional bonding network of the sodium acetate is of vital importance in determining whether high luminescence yields would be obtained.

\section{Interactions with Filter Paper}

Filter paper has been the most widely used solid matrix for RTP. Recently, Hurtubise (7) has reviewed the physicochemical studies related to the RTP of phosphors adsorbed on filter paper. In one set of experiments in this work, the effects of moisture and gases on the RTF and RTP of model aromatic compounds adsorbed on filter paper were studied (8). Water, oxygen, air were investigated for there abilities to quench the RTF and RTP of three model compounds (protonated forms of benzo[f]quinoline and benzo[h]quinoline, and $\alpha$-naphthoflavone). Asis with earlier studies, water was found to be a more potent quencher of RTP than $\mathrm{O}_{2}$. As discussed in section A. of this report, a simple method was developed for reporting the percentage decrease in RTP or RTF for a given quencher. The RTP data were fit to an equation of the form, $\mathrm{Y}=\mathrm{aX} \mathrm{X}^{\mathrm{b}}$, and this equation permitted some conclusions to be made about the interactions responsible for the quenching of RTP by moisture. Presently, a rather detailed model is being developed for the quenching of RTP by water for phosphors adsorbed on filter paper using a comprehensive theory developed by Nissan and co-workers $(9,10)$ for the change in the modulus of paper as a function of moisture. It is planned to more fully develop the model in the future research. Later in this section, our recent work in relating the Nissan model for the change in 
modulus of paper with temperature to phosphorescence lifetimes and phosphorescence quantum yields is outlined.

The effects of $\mathrm{NaF}, \mathrm{NaCl}, \mathrm{NaBr}$, and $\mathrm{NaI}$ on the solid-matrix RTF and RTP of the phenylphenol isomers adsorbed on filter paper were investigated (11). As expected, the RTF signals of the three phenylphenols showed a decrease in intensity with an increase in the molecular weight of the salt. However, due to heavy atom effect, the RTP signals increased substantially with the molecular weight of the salt. Several quenching models were examined for the RTF data. A modified Perrin model was found to represent the data very effectively because it considers the unquenched fluorescence and other aspects related to fluorescence quenching. The results of this work form a basis for modelling RTF quenching on solid matrices and the heavy-atom effect on solid matrices.

The effects of temperature on the photophysical properties of 4-phenylphenol (12) and the protonated form of benzo[f]quinoline $\left(\mathrm{B}[\mathrm{f}] \mathrm{QH}^{+}\right)(13)$ adsorbed on filter paper were obtained. RTF and RTP quantum yields, and phosphorescence lifetimes were acquired. From these parameters, intersystem crossing quantum yields, phosphorescence rate constants, and rate constants for the radiationless transition from the triplet state were calculated. In addition, energy level diagrams were obtained that showed how the radiative and nonradiative energy was distributed in the adsorbed lumiphors. This detailed photophysical information is the first of its type for compounds adsorbed on filter paper and is very important in mechanistic studies. For example, for both model compounds, the phosphorescence quantum yields and intersystem quantum yields were very sensitive to temperature changes. These results indicated that not only phosphorescence quantum yields are important in investigating interactions, but also intersystem quantum yields are important. One of the most important conclusions is that there is a 
relationship between the modulus of paper and phosphorescence quantum yields. This relationship was developed from the concept that modulus is a measure of the rigidity of the matrix, and for high phosphorescence quantum yields, it is necessary for the phosphor to be held rigidly in the matrix. More on the relationship between modulus and phosphorescence parameters is given later in this section. The results from this part of the research revealed the relative complexity of the luminescence processes for solid-matrix luminescence analysis and indicated that several phenomena have to be considered in explaining the interactions for RTF and RTP. The methodology developed will be used "routinely" in the future for additional interaction studies.

In other experiments, the RTF lifetimes of $B[f] Q$ and 4-phenlyphenol adsorbed on filter paper were used with earlier photophysical data to calculate a variety of photophysical parameters for these two model compounds (14). Also, several equations that relate radiative and nonradiative rate constants to pre-exponential factors and activation energy terms were developed. These equations, in turn, were used to derive equations for the two model compounds that relate fluorescence and phosphorescence quantum yields to pre-exponential factors, activation energies, and temperature. The primary importance of this portion of the research is that the approach used to derive the photophysical equations can be used in a general fashion to obtain similar equations for different model compounds adsorbed on solid matrices.

Probably the most exciting advancements in the research during this funding, riod was the development of equations that relate phosphorescence parameters of phosphors adsorbed on filter paper to the Young's modulus of filter paper (15). The experimental values of Young's modulus for filter paper were obtained as a function of temperature. The experimental modulus values for the filter paper samples, along with literature modulus values for regenerated cellulose 
and chromatography paper, were used to derive equations that relate the Young's modulus values of filter paper to the phosphorescence lifetimes of adsorbed phosphors as a function of temperature. In addition, an equation was derived for one of the phosphors that relates the change in phosphorescence quantum yield with temperature to Young's modulus. The basis for the equations is a theory for hydrogen-bond-dominated solids that considers Young's modulus as related to the average force constant for the hydrogen bonds in paper and the effective number of hydrogen bonds in paper $(9,10)$. For all the systems investigated, linear relationships were obtained between $\ln$ (phosphorescence lifetime) and $\ln$ (Young's modulus). Also, a linear equation was obtained between $\ln$ (phosphorescence quantum yield) and $\ln$ (Young's modulus) for one of the phosphors. In general, the results of this work form the basis of a fundamental theory for the phosphorescence of compounds adsorbed on filter paper.

\section{E. Interactions with $\beta$-Cyclodextrin $/ \mathrm{NaCl}$ Matrices}

It was found that $30 \% \beta$-cyclodextrin $(\beta-\mathrm{CD}) / \mathrm{NaCl}$ was a very effective solid matrix for obtaining RTF and RTP from adsorbed compounds (15). The work with $30 \% \beta-\mathrm{CD}$ was an outgrowth of our earlier work with $\alpha-\mathrm{CD} / \mathrm{NaCl}$ mixtures (16-18). In the initial work with $30 \%$ $\beta-\mathrm{CD} / \mathrm{NaCl}$, linear ranges for calibration curves, reproducibility data, and limits of detection were obtained for p-amincbenzoic acid and phenanthrene. Limits of detection for RTF and RTP were in the subnanogram range. Also, the spectra obtained with the $30 \% \beta-\mathrm{CD} / \mathrm{NaCl}$ matrix were practically the same in structural detail as those acquired at low temperature. In addition, the $\beta-\mathrm{CD} / \mathrm{NaCl}$ mixture was very easy to handle for low temperature analysis, and very small amounts of analyte were needed to insure good luminescence spectra (15).

Experiments were carried out to obtain correlations between solution interactions and solid-matrix interactions in $\beta$-CD solid-matrix luminescence (20). These were the first 
experiments of this type, and it is planned to do additional experiments in the future. A variety of liquid chromatographic, solution fluorescence, and extraction data were obtained in an attempt to correlate solution interactions with solid-matrix interactions for solute- $\beta$-cyclodextrin complexes. From the chromatographic data, dissociation constants were calculated for the complexes. In addition, fluorescence data were obtained for sodium chloride, glucose, and $\beta$-cyclodextrin solutions of several solutes. Also, extraction experiments were performed in an attempt to remove the solutes from the $\beta$-CD solid matrix. Generally, the results revealed that there were no simple correlations between the solution data and the solid-matrix luminescence data. However, the extraction results yielded important information related to the solute interactions in the solid matrix. These results indicated that the model compounds were not simply adsorbed onto the surface but were held very tightly in the matrix by complex formation with $\beta-C D$.

Fluorescence and phosphorescence quantum yields, fluorescence and phosphorescence polarization values, and phosphorescence lifetimes were obtained for B[f]Q and 4-phenylphenol adsorbed on $\beta-\mathrm{CD} / \mathrm{NaCl}$ matrices as a function of temperature from 296 to $93 \mathrm{~K}(21,22)$. Several fundamental photophysical parameters were calculated that were correlated with the interactions of $\mathrm{B}[\mathrm{f}] \mathrm{Q}$ and 4-phenylphenol with $\beta-\mathrm{CD} / \mathrm{NaCl}$ matrices and with filter paper (22). In general, the luminescence quantum yields for compounds on $\beta-\mathrm{CD} / \mathrm{NaCl}$ matrices gave higher fluorescence and phosphorescence quantum yields than for similar compounds adsorbed on filter paper. By comparing radiative and nonradiative photophysical rate constants for the different systems studied, it was possible to determine which photophysical processes were enhancing and diminishing the luminescence quantum yields. One of the most amazing results was that the RTP quantum yield of $\mathrm{B}[\mathrm{f}] \mathrm{Q}$ adsorbed on $30 \% \quad \beta-\mathrm{CD} / \mathrm{NaCl}$ was almost the same as the 
phosphorescence quantum yield of $B[f] Q$ obtained at $53 \mathrm{~K}(21)$.

An important development, was the ability to obtain luminescence polarization values as a function of temperature from compounds adsorbed on $\beta-\mathrm{CD} / \mathrm{NaCl}$ matrices $(21,22)$. From this data rotational relaxation times of the phosphors were calculated. Very long rotational relaxation times were obtained for $B[f] Q$ and 4-phenylphenol. These results proved that the phosphors were held extremely tightly to $\beta-\mathrm{CD} / \mathrm{NaCl}$ matrix. Calculated activation energies from the phosphorescence polarization data permitted an important correlation to made with the nonradiative rate constant from the triplet state of 4-phenylphenol. The correlation showed that the nonradiative rate constant for 4-phenylphenol was directly affected by a specific rotational relaxatıon process in the $\beta-\mathrm{CD} / \mathrm{NaCl}$ matrix (22).

\section{F. Analytical Figures of Merit and Analytical Methods of Development}

Several important aspects in analytical methodology were explored. Analytical figures of merit were obtained for the room-temperature luminescence of $B[f] Q$, p-aminobenzoic acid, phenanthrene, and 4-phenylphenol adsorbed on silica gel with polyacrylate binder, filter paper, $1 \%$ polyacrylic acid binder, and $80 \% \alpha$-cyclodextrin/ $\mathrm{NaCl}(23)$. The experimental conditions were optimized to gir'e the "best" luminescence signals for a given system. In some cases, filter paper and $80 \% \alpha$-cyclodextrin $/ \mathrm{NaCl}$ gave comparable analytical results. However, filter paper gave the best overall analytical results with limits of detection in the sub-nanogram range. It should be mentioned that the previous study did not include our recent work with $\beta$-cyclodextrin$/ \mathrm{NaCl}$. As discussed earlier in the report, $\beta$-cyclodextrin $/ \mathrm{NaCl}$ yielded very high luminescence quantum yields from adsorbed compounds.

With the use of four model compounds of widely different functionality, it was found that $1 \% \alpha$-cyclodextrin $/ \mathrm{NaCl}$ mixture could be used in place of an $80 \% \alpha$-cyclodextrin $/ \mathrm{NaCl}$ mixture 
to obtain the same analytical luminescence results as with the $80 \% \alpha$-cyclodextrin $/ \mathrm{NaCl}(24)$. The primary advantages of using $1 \%$ r -cyclodextr:n/ $\mathrm{NaCl}$ are the lower cost and easier handling of the $\alpha$-cyclodextrin $/ \mathrm{NaCl}$ matrices.

The room-temperature luminescence properties of the volarile compounds, 1- and 2naphthol, and four other model compounds adsorbed on filter paper and treated with salts and cyclodextrins were investigated to determine if cyclodextrins would anchor volatile compounds to filter paper and enhance the luminescence of the adsorbed compounds (25). 1-Naphthol and 2-nap'ithol are known to volatilize upon heating along with solvents such as ethanol and water. The filter paper, treated with salts and cyclodextrins, was shown to he capable of trapping these volatile phosphors efficiently on filter paper and permitting strong luminescence signals to be observed. In general, there were enhanced luminescence signals from filter paper treated with $\alpha$-cyclodextrin. For example, the limits of detection for 4-phenylphenol improved from $1.5 \mathrm{ng}$ for both RTF and RTP on untreated paper to 0.65 to $0.75 \mathrm{ng}$ for RTF and RTP, respectively.

$\beta$-Cylcodextrin/salt mixtures were investigated as solid matrices for the RTF and RTP of four tetrols. Tetrols are degradation products from tenzo(a)pyrene-DNA adducts and are stereoisomeric in nature (26). The luminescence spectral properties of the four tetrols showed similar properties when adsorbed on $\beta$-cyclodextrin/salt mixtures. Several heavy-atom salts were investigated for enhancing the RTP of the tetrols. However, there was only a moderate increase in the phosphorescence from the tetrols with a heavy atom present. Generally, $\beta$-cyclodextrin$\mathrm{NaCl}$ matrices were very effective in yielding phosphorescence from the tetrols. The RTP lifetimes of the adsorbed tetrols showed a wide range of values which depended on the characteristics of the solid-matrices. From the RTP lifetime results a sensitive RTP time-resolved phosphorescence method at the nanogram level was developed for a mixture of two of the ietrols. 
G. Reversed-Phase Liquid Chromatography with $\beta$-Cyclodextrin as a Mobile-Phase Modifier

Experiments were carried out to determine the effects of $\beta$-cyclodextrin on the retention characteristics of hydroxyl aromatics in reversed-phase liquid chromatography. This work was partially supported by the DOE grant $(27,28)$. This type of research is important in understanding the solution interactions in the sample preparation step in cyclodextrin solid-matrix luminescence. In earlier work, we discussed the mechanistic aspects of retention with $\beta$-cyclodextrin present in reversed-phase mobile phases (29). We expanded that research under this grant to obtain the changes in enthalpy and entropy of hydroxy! aromatics in reversed-phase liquid chromatography with $\beta$-cyclodextrin in the mobile phase (27). From the thermodynamic data, it was shown that the hydroxyl aromatics investigated could be grouped into two classes, and different interactions were operative with the two groups of hydroxyl aromatics. In a practical application, it was shown that very substantial improvements were obtairied for the separation of hydroxyl aromatics with $\beta$-cyclodextrin added to the mobile phase (28).

\section{H. Manuscripts and Presentations}

The manuscripts published, in press, or in review that were written under this project are listed on pages 14-16 in this report. These are references 1, 4-8, 11-16, and 20-26. References 27 and 28 were written with the partial support of DOE funds. Several other manuscripts are in preparation. A total of seventeen presentations of the work under this project were given at various meetings by principal investigator, postdoctoral associate, and graduate students.

\section{REFERENCES}

1. Citta, L.A.; Hurtubise, R.J. Talanta, in review, "Analytical and Mechanistic Aspects of the Room-Temperature Fluorescence and Phosphorescence of Benzo[f]qinoline Adsorbed on Silica gel Chromatoplates with Humidified Gases". 
2. Ramasamy, S.M.; Hurtubise, R.J. Anal. Chem., 1987, 59, 432, "Effects of Temperature on the Solid-Surface Luminescence Properties of p-Aminobenzoic Acid Adsorbed on Sodium Acetate".

3. Ramasamy, S.M.; Hurtubise, R.J. Anal. Chem., 1987, 59, 2144, "Room-Temperature Luminescence Properties of p-Aminobenzoic Acid Adsorbed on Sodium Acetate-Sodium: Chloride Mixtures".

4. Ramasamy, S.M.; Hurtubise, R.J. Appl. Spectrosc.,1990, 44. 1494, "Physicocheritical Interactions of the Isomers of Aminobenzoic Acid with Sodium Acetate in Solid-Matrix Room-Temperature Luminescence Spectroscopy".

5. Ramasamy, S.S1.; Hurtubise, R.J. Anal. Chem., 1990, 62, 1060, "Comparative Study if Solid-Matrix Luminescence Interactions of p-Aminobenzoate on Two Different Matrices".

6. Ramasamy, S.M.; Hurtubise, R.J. Appl. Spectrosc. in press, "Solid-Matrix Luminescence Polarization of p-Aminobenzoate Adsorbed on Sodium Aceiate and Sodium Acetate/Sodium Chloride Mixtures".

7. Hurtubise, R.J. "Phosphorimetry: Theory, Instrumentation, and Applications"; VCH Publishers Inc.: New York, 1990.

8. Citta, L.A.; Hurtubise, R.J. Appl. Spectrosc., in press, "The Effects of Moisture and Gases on the Room-Temperature Fluorescence and Phospiorescence of Model Aromatic Compounds Adsorbed on Filter Paper".

9. Batten, G.L.; Nissan, A.H. Tappi J. 1987, 70, 119, "Unified Theory of the Mechanical Properties of Paper and Other H-Bond-Dominated Solids-Part I".

10. Nissan, A.H.; Batten, G.L. Tappi J., 1990, 73, 159, "On the Primacy of the Hydrogen Bond in Paper Mechanics".

11. Purdy, B.B.; Hurtubise, R.J. Anal. Chim. Acta, in press, "Effects of Salts on the SolidMatrix Luminescence of Phenylpheno' Isomers Adsorbed on Filter Paper".

12. Ramasamy, S.M.; Hurtubise, R.J. Talan'a, 1989, 36, 315, "Temperature Effects on the Solid-Matrix Luminescence Properties of 4 -Phenylphenol Adsorbed on Filter Paper".

13. Ramasamy, S.M.; Hurtubise, R.J. Appl. Spectrusc., 1989, 43, 616, "The Effects of Temperature on the Solid-Surface Luminescence Properties of Benzo[f]quinoline".

14. Hurtubise, R.J.; Ramasamy, R.J. Appl. Spectrosc., 1991, 45, 555, "Kinetics Aspects in Solid-Matrix Luminescence for Lumiphors Adsorbed on Filter Paper".

15. Hurtubise, R.J.; Ramasamy, S.M. Appl. Spectrosc. 1991, 45, 1126, "An Interaction Model for Solid-Matrix Phosphorescence with Filter Paper via the Modulus of Filter Paper". 
16. Richmond, M.D.; Hurtubise, R.J. Anal. Chem., 1984, 01, 2643, "Analytical Characteristics of $\beta$-Cyclodextrin/Sait inixtures in Room-Temperature Solid-Surface Luminescence Analysis".

17. Bello, J.M.; Hurtubise, R.J. Anal.Clem. 1987, 59, 2395, "Interactions of Compounds Adsorbed on an $80 \% \alpha$-Cyclodextrin-Sodium Chloride Mixture Investigated by Diffuse Reflectance and Luminescence Spectrometry".

18. Belio, J.M.; Hurtubise, R.J. Anal. Chem. 1988, 60, 1291, "Room-Temperature Luminescence Properties of Benzo[f]quinoline and Phenanthrene Adsorbed on $\alpha$-Cyclodextrin-Sodium Chloride Mixtures".

19. Bello, J.M; Hurtubise, R.J. Anal. Chem., 1988, 42, 619, "Room-Temperature Luminescence Properties of p-Amonobenzoic Acid and 4-Phcnylphenol Adsorbed on $\alpha$-Cyclodextrin/ $\mathrm{NaCl}$ Mixtures".

20. Richmond, M.D.; Huctubise, R.J. Talanta, 1990, 37, 1057, "Solution Interactions and SolidMatrix Interactions in $\beta-C$ jclodextrin Solid-Matrix Luminescence".

21. Richmond, M.D.; Hurtubise, R.J. Anal. Chem., 1991, 63, 169, "Luminescence Properties of Benzo[f]quinoline Adsorbed on $\beta$-Cyclodextrin/Sodium Chloride as a Function of Temperature".

22. Richmond, M.D.; Hurtubise, R.J. Anal. Chem. 195.1, 63, 1073, "Physicochemical Inter-.tions of 4-Phenylphenol as a Function of Temperature with $\beta$-Cyclodextrin/Sodium Chloride Solid Matrices".

23. Purdy, B.B.; Hurtubise, R.J. Microchem. J., 1989, 39, 330, "Room-Temperature Luminescence of Benzo[f]quinoline, p-Aminobenzoic Acid, Phenanthrene, and 4-Phenylphenol on a Variety of Solid Surfaces".

24. Richmond, M.D.; Hurtubise, R.J. Appl. Spectrosc., 1989, 43, 810, "Room-Temperature Luminescence of a Variety of Compounds on $1 \% \alpha$-Cyclodextrin/ $\mathrm{NaCl}$ Mixture".

25. Ramasamy, S.M.; Hurtubise, R.J. Microchem. J., 1989, 40, 317, "Room-Temperature Luminescence of Volatile Compounds Absorbed on Filter Paper 'Treated with Salts and Cyclodextrins".

26. Richmond, M.D.; Hurtubise, R.J. Anal. Chim. Acta, in press, " $\beta$-Cyclodextrin Solid-Matrix Luminescence Characterization of Stereoisomeric Tetrols".

27. Mohseni, R. M.; Hurtubise, R.J. J. Chromatogr. 1991, 537, 61, "Changes in the Enthalpy and Entropy of Hydroxyl Aromatics in Reversed-Phase Liquid Chromatography with $\beta$-Cyclodextrin in the Mobile Phase". 
28. Mohseni, R. M.; Hurtubise, R.J. J. Chromatogr., 199(), 514, 19, "Reversed-Phase Chromatographic Separation of Selected Hydroxyl Aromatics with $\beta$-Cyclodextrin as a Mohile Phase Additive".

29. Mohseni, R.M.; Hurtubise, R.J. J.Chromatogr. 1990, 499, 395, Retention Characteristics of Several Compound Classes in Reversed-Phase Liquid Chromatography with $\beta$-Cyclodextrin as a Mobile Phase Modifier". 

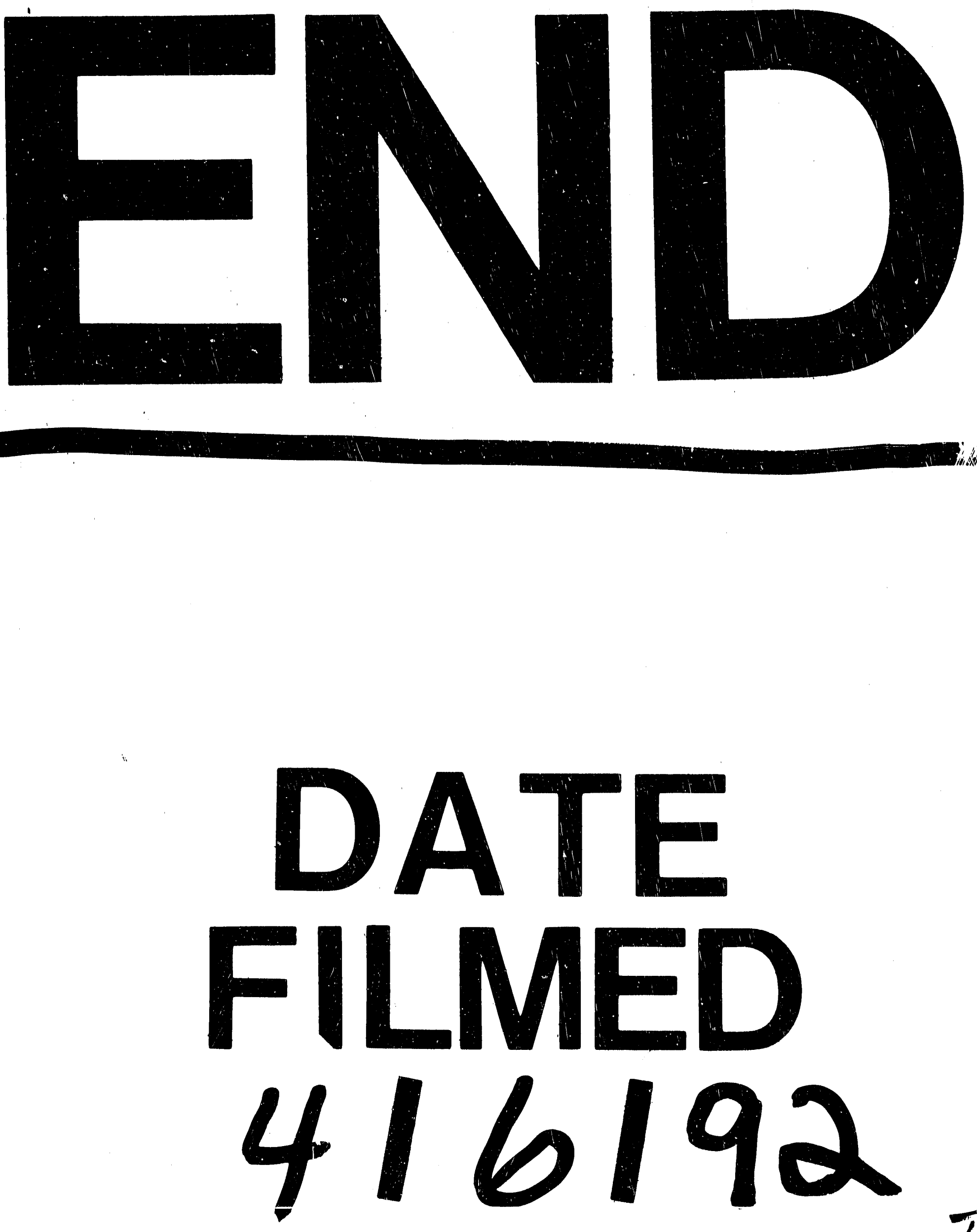

$I$ 
